\title{
La diversidad cultural desde la perspectiva de los/as educadores/ as sociales: un estudio en los centros educativos de Andalucía
}

\section{Cultural diversity from the perspective of social educators: a study in schools in Andalusia}

\author{
Teresa Terrón-Caro ${ }^{1}$ \\ mttercar@upo.es \\ Teresa Rebolledo Gámez \\ tjrebgam@upo.es \\ M. ${ }^{a}$ Del Rocío Rodríguez Casado \\ mrrodcas@upo.es \\ Macarena Esteban Ibáñez \\ mestiba@upo.es \\ Universidad Pablo de Olavide, España
}

\section{Resumen:}

En España, la atención a la diversidad cultural constituye uno de los retos socioeducativos al que la escuela se enfrenta. La presencia del alumnado de origen extranjero, entre otras causas, ha evidenciado que se hacen necesarias medidas de actuación renovadas que atiendan esta realidad. Tomando como referencia a la Comunidad Autónoma Andaluza, en las siguientes líneas se plantea un estudio cuyo objetivo es analizar las actuaciones de los centros educativos para gestionar la diversidad cultural, desde el punto de vista de los/as educadores/as sociales u otros agentes educativos que son responsables de su desarrollo. Para ello, se ha utilizado una metodología de carácter cuantitativo,

\begin{abstract}
:
1. In Spain, attention to cultural diversity is one of the socioeducational challenges that school confront. The presence of immigrant students, among other things, has shown that it is necessary to undertake updated measures to attend this reality. Within the Andalusian context, this study aims to analyse how schools have managed cultural diversity, from the point of view of social educators or other educators in charge of inclusion programs. To do this, we have used a quantitative methodology based on a questionnaire designed for this research, which was distributed to all schools in Andalusia which had incorporated the figure of the social educator in the academic year 2009-10.
\end{abstract}

1 Dirección para correspondencia (correspondence address):

Teresa Terrón-Caro. Dpto. de Educación y Psicología Social. Facultad de Ciencias Sociales. Universidad Pablo de Olavide. Carretera de Utrera, Km. 1, edificio 11. 41013, Sevilla (España). 
La diversidad cultural desde la perspectiva de los/as educadores/as sociales: un estudio en los centros educativos de Andalucía

Teresa Terrón-Caro, Teresa Rebolledo Gámez, M. a del Rocío Rodríguez Casado y Macarena ESTEBAN IBÁÑ̃EZ

implementando un cuestionario diseñado para la presente investigación, el cuál se hizo llegar a todos los centros educativos de Andalucía en los que se había incorporado la figura del/a Educador/a Social en el curso 2009-10 y la información recogida ha sido tratada estadísticamente. Los resultados obtenidos apuntan una gran complejidad en el análisis de la gestión de la diversidad en las escuelas andaluzas, donde no se muestran referentes claros en los modelos de actuación. Se defiende que las prácticas con perspectivas interculturales pueden favorecer la lucha contra las desigualdades, la participación de la comunidad educativa y la inclusión del alumnado diverso, avanzando a una mejora de la calidad educativa para todos los miembros implicados.

\section{Palabras clave:}

Educador/a social; diversidad cultural; escuela; inclusión.
The information gathered has been treated statistically. The results point to a high complexity in the analysis of diversity management in Andalusian schools since no clear unitary points of reference in all the models could be found. It is argued that intercultural actions can foster the fight against inequalities, participation of the teaching community and the inclusion of diverse students, which surely leads to improving the quality of education for all the members involved.

\section{Key words:}

Social educator; cultural diversity; school; inclusion.

\section{Résumé:}

En Espagne, I'attention à la diversité culturelle est l'un des défis socioéducatifs auquelle I 'école est confrontée. La présence des élèves immigrants, entre autres, a montré qu'il est nécessaire d'avoir des mesures renouvelées qui traitent cette action en réalité. En prenant comme référence l>Andalousie, dans les lignes suivantes on soulève une étude visant à analyser les performances des écoles à gérer la diversité culturelle, du point de vue des éducateurs sociaux ou d`autres agents éducateurs qui sont responsables de leur développement. Pour cela, nous avons utilisé une méthode quantitative, la mise en œuvre d)un questionnaire conçu pour cette recherche, qui a été distribué à toutes les écoles de 1)Andalousie et dans lequel a été incorporé la figure du éducateur social pour l'année scolaire 2009-10 et l'informations recueillie a été traitée statistiquement. Les résultats obtenus font état d`une grande complexité dans l sanalyse de la gestion de la diversité dans les écoles andalouses où les références claires ne sont pas présentées dans les modèles d'action. II fait valoir que la pratique des perspectives interculturelles peut favoriser la lutte contre les inégalités, la participation de la communauté éducative et lsinclusion de divers élèves, en améliorant la qualité de liéducation pour tous les membres impliqués.

\section{Mots clés:}

Éducateur social; diversité culturelle; école; inclusion.

Fecha de recepción: 29-7-14

Fecha de aceptación: 10-2-2015 
Teresa Terrón-Caro, Teresa Rebolledo Gámez, M. ${ }^{a}$ Del Rocío Rodríguez Casado y Macarena

\section{Introducción}

Este trabajo presenta los resultados obtenidos en el proyecto de investigación denominado "Atención a la diversidad cultural en instituciones educativas" ${ }^{2}$, en el que se plantea como finalidad conocer la gestión de la diversidad cultural en los centros educativos de la Comunidad Autónoma Andaluza, desde el punto de vista de los/as educadores/as sociales como agentes de la intervención en las escuelas.

La atención a la diversidad cultural constituye uno de los retos fundamentales que siguen afrontando las escuelas en la actualidad. En España, entre otros aspectos, la presencia progresiva de alumnado extranjero en las aulas ha obligado a que la diversidad sea uno de los elementos a tener en cuenta en las políticas de inclusión de los últimos años (Rodríguez Navarro et al., 2011). Si bien la forma en que las políticas sociales y educativas deben abordar esta pluralidad, así como la necesidad de sensibilización y fomento de una educación intercultural dirigida a toda la comunidad educativa, es, hoy en día, una de las principales ocupaciones de todos aquellos implicados en la enseñanza.

Es conocido que, desde los años 90 del pasado siglo, España se ha consolidado como uno de los destinos preferentes de las migraciones motivadas por la búsqueda de mejores condiciones de vida, generando la convivencia o coexistencia de diferentes culturas en áreas geográficas concretas. Ello ha permitido tratar la educación intercultural como objeto de reflexión y debate desde diferentes disciplinas educativas y sociales (Álvarez, 2008).

Según datos de la Secretaria General de Inmigración y Emigración (Ministerio de Educación, Cultura y Deporte, 2014), la evolución del número de personas extranjeras residentes en España se ha incrementado en más de un $300 \%$ en los últimos diez años, si bien es cierto que desde 2008, con la crisis económica, se apunta una disminución en los porcentajes de crecimiento, alcanzando la cifra de 5.503.977 a junio de 2013.

De esta realidad se desprende la exigencia a la que se somete nuestra sociedad para solventar los cambios acontecidos en las necesidades y demandas que se plantean con respecto a la inmigración en los diferentes ámbitos (sociales, culturales, políticos, económicos, religiosos, etc.),

2 Investigación enmarcada en el Grupo Emergente de Investigación SEJ-457, financiado por la Universidad Pablo de Olavide, de Sevilla (Resolución 08/13-APP de 30 de julio de 2008). 
La diversidad cultural desde la perspectiva de los/as educadores/as sociales: un estudio en los centros educativos de Andalucía

Teresa Terrón-Caro, Teresa Rebolledo Gámez, M. ${ }^{a}$ del Rocío Rodríguez Casado y Macarena ESTEBAN IBÁÑ̃z

a la vez que se convierte en una realidad que nos permite convivir en permanente contacto con otras culturas (Schramkowski, 2008).

En lo referente al ámbito escolar, este escenario también se refleja en las cifras de alumnado extranjero matriculado en las aulas españolas.

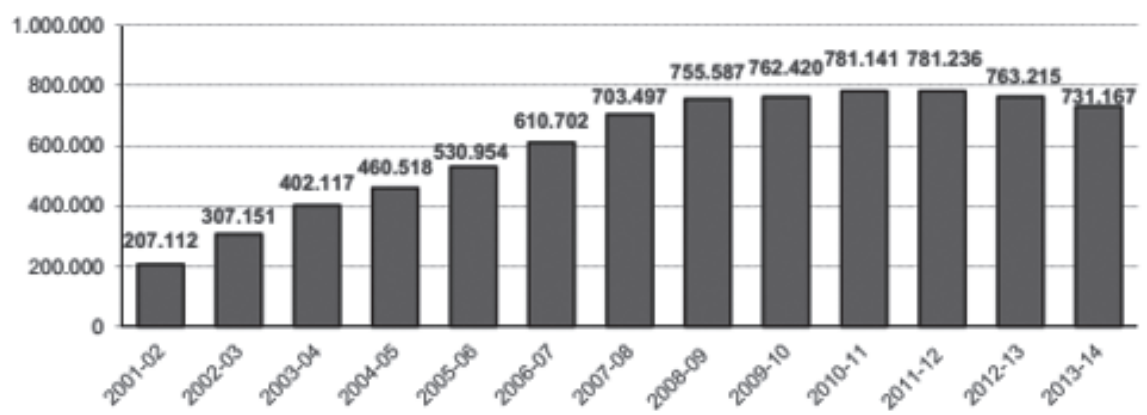

Figura 1. Evolución del alumnado extranjero matriculado en enseñanzas no universitarias.

Fuente: Ministerio de Educación, Cultura y Deporte, 2014.

Como se apunta en la Figura 1, los datos de alumnado extranjero matriculado en las enseñanzas no universitarias han sufrido un crecimiento permanente hasta el curso 2008-2009, donde comienza un incremento más comedido en comparación con el periodo anterior. A partir del curso 2012-2013, finalmente se ha desembocado en una reducción de la cifra total de alumnado.

Sin embargo, se debe tener presente que, atendiendo a la proporción con respecto al resto del alumnado, el alumnado extranjero del curso 2013-2014 supone un 9.05\% del conjunto total (Ministerio de Educación, Cultura y Deporte, 2014), cifra relevante para comprender su importancia dentro del ámbito educativo.

A nivel autonómico, la realidad encontrada es similar a la estatal. En Andalucía, los datos de los últimos cursos también son bastantes estables, con un crecimiento moderado del alumnado extranjero, cuya representación es de un 5.2\% en relación a la totalidad del alumnado matriculado en el curso 2013-2014 (Ministerio de Educación, Cultura y Deporte, 2014).

Esta realidad con respecto a las escuelas, hace necesaria la implantación de medidas renovadas e innovadoras que atiendan a la diversidad cultural en los centros escolares, con el fin de garantizar la lucha contra 
Teresa Terrón-Caro, Teresa Rebolledo Gámez, M. ${ }^{a}$ del Rocío Rodríguez Casado y Macarena

las desigualdades y una educación de calidad para todo el alumnado. Pues, tal y como expone Álvarez (2008, p. 203)

Si la educación (...) es una pieza clave del funcionamiento de las sociedades contemporáneas y un sector prioritario de la política de un país, la educación intercultural es un pilar de la dinámica de las sociedades diversas y de la política democrática de los estados.

En la actualidad, las políticas de inclusión para las escuelas siguen planteando la interculturalidad como modelo a seguir en la intervención. Sin embargo, es difícil encontrar en la práctica real el desarrollo y funcionamiento de esta forma de gestionar la diversidad cultural en las aulas (Rodríguez Navarro et al., 2011). Contrariamente, es frecuente encontrarse en el ámbito escolar la gestión de la diversidad cultural abordada desde otros modelos a considerar, como son los compensatorios, los asimilacionistas o los multiculturales (De Miguel, 1995; Aguado, 2003; Barandica, 2006; Ariño, 2009; Rodríguez Vargas, 2012; Osuna, 2012). Con la idea de aclarar los mencionados modelos, se toma como referencia la clasificación propuesta por Bartolomé et al. (1997), que se basa en el criterio de finalidad de éstos. De esta forma, esta autora, los distinguen en cinco tipos:

1.- El modelo asimilacionista, compensatorio y segregacionista en el que se mantiene la cultura hegemónica de la sociedad donde se sitúa.

2.- El modelo de currículum multicultural y pluralismo cultural en el que se reconoce que existe una sociedad multicultural.

3.- El modelo de orientación multicultural, intercultural, holístico y de educación no racista en el que se fomenta la reciprocidad y solidaridad entre diferentes culturas.

4.- El modelo antirracista y radical con el que se denuncia la injusticia que se deriva de una asimetría cultural y lucha contra ella.

5.- El modelo de proyecto educativo global en el que se avanza hacia un proyecto global educativo que defiende la interculturalidad y la lucha contra la discriminación.

Llevados a la práctica, por tanto, en los modelos más cercanos a los del primer tipo (asimilacionista, segregacionista, compensatorio), se atribuirá al alumnado extranjero desventajas con respecto al alumnado 
La diversidad cultural desde la perspectiva de los/as educadores/as sociales: un estudio en los centros educativos de Andalucía

Teresa Terrón-Caro, Teresa Rebolledo Gámez, M. ${ }^{a}$ del Rocío Rodríguez Casado y Macarena Esteban IBÁÑez

autóctono, olvidando sus peculiaridades identitarias y entendiendo las mejoras en tanto se acerquen al ideal planteado, que no es otro, que el de la cultura mayoritaria.

Asimismo, modelos similares a la opción multicultural, centrarán sus esfuerzos en el conocimiento de las culturas en aspectos parciales dentro del marco escolar, como es el caso del currículo, el cual puede modificarse parcialmente para introducir contenidos culturales, pero manteniendo una perspectiva estereotipada o folclórica de las culturas minoritarias.

Por último, la gestión de la diversidad basada en la interculturalidad, defiende la simetría cultural, entendida como el respeto a la diversidad cultural, a la promoción de la comunicación y el diálogo entre los diferentes grupos culturales, cuya finalidad es Ilegar a todo el alumnado, primando la solidaridad y la reciprocidad entre culturas.

A diferencia del resto de modelos, la educación intercultural se dirige a todo el alumnado, sin distinción por su procedencia, así como se enmarca en todos los centros, no sólo en aquellos con presencia de minorías étnico-culturales. Así, diferentes autorías como De Miguel (1995), Besalú (2002), Aguado (2004), Ortega (2004), Barandica (2006) y Osuna (2012), resaltan la importancia de que los centros escolares se adapten, modifiquen e innoven para atender las necesidades y demandas de nuestra sociedad, trabajando una interculturalidad centrada en principios como el conocimiento, compresión y respeto mutuo de las diferentes culturas, el diálogo y solidaridad como valor positivo y proyecto de intercambio para un enriquecimiento recíproco, la igualdad de oportunidades, la lucha contra la discriminación y el respeto por los derechos humanos.

De esta forma, la interculturalidad posee multitud de dilemas morales y éticos en el ámbito socioeducativo donde hay que realizar un exhaustivo trabajo de información y formación, así como de enseñanzaaprendizaje en educación intercultural. Estos factores giran en torno al desarrollo y fomento de la equidad educativa como principio; al progreso contra el racismo, dando posibilidad y facilitando la comunicación y competencia interculturales y, en general, a la promoción de un cambio en la sociedad hacia un mundo más justo y democrático en valores socioeducativos (Gil-Jaurena, 2013).

En este sentido, ante esta realidad, en el Plan Estratégico de Ciudadanía e Integración de España (2007-2010, p.225) se expone que 
Teresa Terrón-Caro, Teresa Rebolledo Gámez, M. ${ }^{a}$ Del Rocío Rodríguez Casado y Macarena

(...) una gestión efectiva de la interculturalidad requiere del sistema educativo el impulso del conocimiento, la percepción y la comprensión de grupos y culturas distintos a los propios de tal modo que, la búsqueda de las semejanzas y la comprensión de las diferencias, logran una convivencia enriquecedora.

Además, hay que considerar que en la gestión de la diversidad cultural de las escuelas todos los agentes de la comunidad educativa se encuentran implicados, desde el profesorado, destacado por su relación directa con el alumnado, hasta las familias, pasando por los equipos directivos y otros agentes internos y externos. De acuerdo con Rodríguez Navarro et al. (2011, p. 108) la participación de la comunidad educativa conlleva el mantenimiento de las "estructuras cooperativas y dialógicas, a resolver conflictos, a atender a la lengua, a realizar una acogida de forma global. En este sentido, la participación se busca dentro de la familia, del profesorado y de diferentes agentes sociales".

Con la finalidad de atender a la diversidad cultural en los centros escolares, en Andalucía, la Ley 17/2007, de 10 de Diciembre, de Educación de Andalucía, deposita un papel fundamental del desarrollo de las acciones de atención a la diversidad cultural sobre el espacio de la orientación escolar. Dentro de dicho ámbito, como principales apoyos externos a las escuelas, sobre los que recae gran parte de la responsabilidad de atender a las realidades socio-culturales, se encuentran los Equipos de Orientación Educativa (en adelante, EOEs). Dichos equipos se caracterizan por la interdisciplinariedad y especialización de sus actuaciones, por su función de apoyo a la actividad pedagógica, por su perspectiva de zona y contribución a la dinamización pedagógica e innovación educativa (Decreto 213/1995, de 12 de Septiembre, por el que se regulan los Equipos de Orientación Educativa).

Los EOEs son unidades básicas de orientación pedagógica que, mediante el desempeño de funciones especializadas en las áreas de orientación educativa, atención al alumnado con necesidades educativas especiales, compensación educativa y apoyo a la función tutorial del profesorado, actúan en el conjunto de los centros de una zona educativa, cuya responsabilidad recae en la Consejería de Educación.

La división de zonas educativas se realiza en base a los criterios de número de alumnado por etapas educativas, características geográficas del lugar y características sociales y educativas más relevantes de la po- 
La diversidad cultural desde la perspectiva de los/as educadores/as sociales: un estudio en los centros educativos de Andalucía

Teresa Terrón-Caro, Teresa Rebolledo Gámez, M. ${ }^{a}$ del Rocío Rodríguez Casado y Macarena Esteban IBÁÑez

blación escolar. Esta designación se produce con la intención de favorecer la coordinación y el trabajo conjunto entre todos los profesionales que actúan en el mismo contexto escolar (Decreto 213/1995, de 12 de Septiembre, por el que se regulan los Equipos de Orientación Educativa).

Cabe destacar que los EOEs, a excepción de casos particulares, desarrollan su labor en centros educativos públicos (Orden de 23 de julio de 2003, por la que se regulan determinados aspectos sobre la organización y el funcionamiento de los Equipos de Orientación Educativa). Su intervención se gestiona a través de la Jefatura de Estudios o, en el caso de los Institutos de Educación Secundaria (IES), en colaboración de los Departamentos de Orientación. En todo caso, es la Jefatura de Estudios del centro la que deberá garantizar que los EOEs dispongan de espacios y recursos adecuados para desarrollar sus labores.

Con respecto a su disposición profesional, inicialmente, se componía por psicólogos, pedagogos, médicos y maestros y, en caso de necesidad por las características de la zona, por trabajadores sociales. Sin embargo, normativas posteriores han ido regulando la incorporación de otros tipos de profesionales que, por sus especialidades, eran demandados en las actuaciones que se realizaban. Es el caso de los/as educadores/ as sociales, que si bien venían actuando dentro de estos equipos desde hace algunos años, es desde la publicación de las "Instrucciones de la Dirección General de Participación e Innovación Educativa por las que se regula la intervención del educador y educadora social en el ámbito educativo" (Junta de Andalucía, Consejería de Educación, 2010), cuando se regula sus funciones y competencias en el ámbito escolar.

Por su corta trayectoria, la figura del Educador/a Social se encuentra en continuo proceso de desarrollo, avanzando como agente social que interviene en el ámbito educativo para facilitar y posibilitar que la ciudadanía logre la plena integración social participando activamente en su comunidad. Como agente socioeducativo inmerso en las escuelas y que interviene ante la diversidad cultural presente, el educador/a social debe poseer competencias interculturales para un mayor y mejor desempeño profesional en dicho contexto (Terrón-Caro y Cárdenas-Rodríguez, 2012).

Esta misma autoría defiende las palabras de Aneas (2005) que razona aludiendo que una persona con competencias interculturales se considera "hábil, eficiente o apta para desarrollar sus tareas y funciones en contextos profesionales multiculturales". Por otra parte, profundiza que 
dichas competencias interculturales conforman todo un abanico de "requisitos, actitudes, destrezas, conocimientos que son los que permiten (...) dar respuesta adecuada a los requerimientos de tipo profesional, interpersonal y afectivo que emergen de los citados contextos multiculturales" (Terrón-Caro y Cárdenas-Rodríguez, 2012, p. 34).

En definitiva, la educación social engloba diferentes campos disciplinares, como son la antropología, la psicología, la sociología, el derecho, la ética,... para analizar, diagnosticar, intervenir y evaluar una determinada realidad socioeducativa con el objeto de mejorarla o transformarla, dándole especial protagonismo a los propios destinatarios. Es decir, tal y como expone Sáez y Campillo (2013) la relación existente entre el ámbito social y educativo favorece en cierta medida, siempre que se planteen de forma adecuada, el campo pedagógico y su profesionalización.

Partiendo de estas premisas, en este estudio se realizan las siguientes preguntas de investigación: ¿De qué manera se atiende a la diversidad cultural en los centros educativos andaluces? ¿Cuál es el enfoque de atención a la diversidad cultural que tienen los centros educativos andaluces? ¿Qué medidas Ilevan a cabo para atender al alumnado de origen extranjero? ¿Qué recursos se ponen a disposición de las familias de origen extranjero para facilitar su relación con los centros? ¿Qué apoyo proporciona la Administración pública a los centros para atender a la diversidad cultural?

\section{Métodos}

\subsection{Objetivos}

El objetivo general de este estudio es analizar las actuaciones que los centros educativos públicos de la Comunidad Autónoma de Andalucía desarrollan para gestionar la diversidad cultural, desde el punto de vista de los/as educadores/as sociales u otros agentes educativos que son responsables de su aplicación. Como objetivos específicos, se encuentran los siguientes:

- Profundizar en las acciones, medidas y recursos que los centros educativos utilizan para atender a la diversidad cultural de la comunidad educativa, haciendo especial referencia al alumnado como destinatario. 
La diversidad cultural desde la perspectiva de los/as educadores/as sociales: un estudio en los centros educativos de Andalucía

Teresa Terrón-Caro, Teresa Rebolledo Gámez, M. ${ }^{a}$ del Rocío Rodríguez Casado y Macarena ESTEBAN IBÁÑ̃z

- Examinar las relaciones que el centro educativo desarrolla con las familias y agentes del entorno, como forma de gestionar la diversidad cultural presente en el centro educativo.

\subsection{Participantes}

La muestra estaba integrada por el $61.1 \%$ de mujeres y el $38.9 \%$ de hombres, de los/as cuales el $67.6 \%$ eran educadores/as sociales y estaban contratados/as como tales, si bien el $24.3 \%$ poseía una licenciatura -normalmente psicopedagogía-. El resto de sujetos que integraban la muestra eran: profesionales de la pedagogía o psicología que ejercían como orientadores/as del centro $(21.6 \%)$ y/o ostentaban la jefatura de estudio (2.7\%); trabajadores/as sociales que actuaban como tales $(2.7 \%)$, maestros/as que ocupaban la jefatura de estudios (2.7\%) y otros $(2.7 \%)$. En todo caso, las personas que componían la muestra estudiada eran las encargadas o formaban parte del equipo que gestionaba la diversidad cultural en los centros educativos asignados.

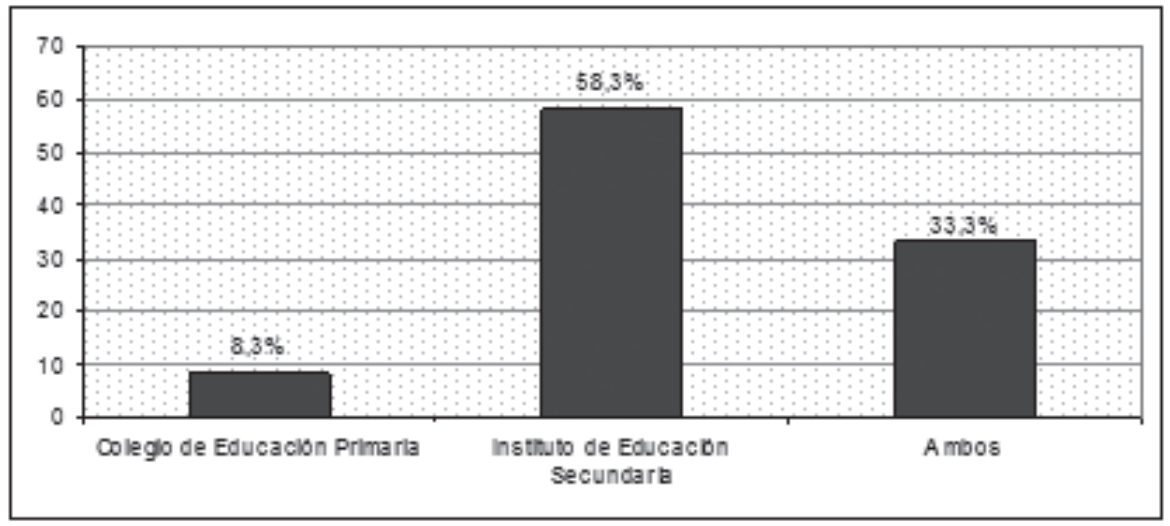

Figura 2. Centro educativo donde trabaja actualmente.

Tal y como se ve reflejado en la figura 2, la mayoría de los/as profesionales trabajaban en Institutos de Educación Secundaria (58.3\%) o en centros de Educación Primaria y Secundaria (33.3\%). Solo un 8.3\% trabajaban exclusivamente en Colegios de Educación Primaria. Estos datos confirman que gran parte de estos/as profesionales pertenecían a equipos que desarrollaban su labor en varios centros de la zona a la que pertenece, independientemente que sean de primaria o secundaria. Asi- 
mismo, es destacable el hecho de que muchos intervenían en los ciclos educativos pertenecientes a la secundaria, con las características que conllevan estas etapas por motivos de edad. La distancia cultural, que puede ser irrelevante en la infancia, se manifiesta en mayor medida en la adolescencia (Carabaña, 2007, citado en García et al., 2008). Debido a ello, pueden aparecer problemáticas en la convivencia derivadas de la presencia de diferencias más significativas entre el alumnado, destacando entre éstas, las originadas por la diversidad étnico-cultural.

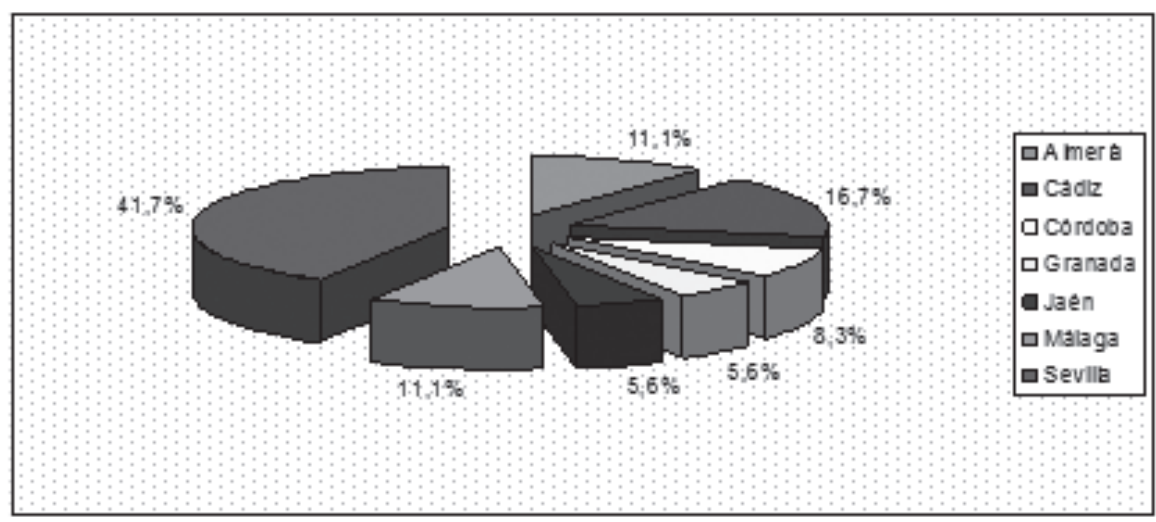

Figura 3. Provincia donde se ubica.

Como información que puede contribuir a comprender el contexto donde se desarrolla esta investigación, se observa en la figura precedente que la muestra del estudio pertenecía a siete de las ocho provincias andaluzas, destacándose Sevilla, representada en el $41.7 \%$, seguido de Cádiz $(16.7 \%$ ) y Almería y Málaga (ambos con un $11.1 \%$ ). Esta distribución de la ubicación de los/as agentes educativos es significativa en tanto puede aportar una aproximación al apoyo de personal que las administraciones públicas proporciona a los centros escolares, independientemente del porcentaje de alumnado extranjero matriculado.

\subsection{Instrumentos de recogida de la información}

La metodología de esta investigación es cuantitativa, utilizando como técnica de recogida de datos la encuesta, cuyo instrumento es el cuestionario.

El cuestionario que ha sido diseñado para esta investigación está in- 
La diversidad cultural desde la perspectiva de los/as educadores/as sociales: un estudio en los centros educativos de Andalucía

Teresa Terrón-Caro, Teresa Rebolledo Gámez, M. ${ }^{a}$ del Rocío Rodríguez Casado y Macarena Esteban IBÁÑez

tegrado por tres dimensiones: centro educativo, funciones y competencias del/la educador/a social para la atención a la diversidad cultural y formación de los/as profesionales educativos en el ámbito de la diversidad cultural. En este artículo nos centraremos en la primera dimensión estudiada, destinada a cuestiones relativas a las actuaciones del centro educativo para la atención a la diversidad cultural. Entre las cuestiones que se desarrollan se encuentran: la presencia de un enfoque intercultural en la documentación oficial de la institución escolar, las medidas específicas que se llevan a cabo en relación a la diversidad cultural, las actuaciones de compensación educativa, tanto ordinales como complementarias y la relación del centro y su coordinación con agentes externos, tales como el órgano competente en materia de educación a nivel autonómico.

En general, esta dimensión se compone de un total de 15 preguntas, siendo su tipología de respuestas cerradas de opción única y opción múltiple y preguntas de respuestas abiertas.

\subsection{Procedimiento}

La validación del instrumento se ha realizado por dos vías: revisión por expertos/as relevantes en metodología y la temática a tratar; y a través de un estudio piloto. Gracias a este procedimiento, se depuró dicho instrumento $y$, tras las modificaciones oportunas, se obtuvo el cuestionario final que se ha utilizado en la investigación. En cuanto a su fiabilidad, el instrumento final tiene un valor de alfa de cronbach de 0.768 .

En el proceso de recogida de datos, los cuestionarios se hicieron llegar a todos los centros educativos de Andalucía donde se había incorporado la figura del/a Educador/a Social tras la publicación de las "Instrucciones de la Dirección General de Participación e Innovación Educativa por las que se regula la intervención del educador y educadora social en el ámbito educativo" (Junta de Andalucía, Consejería de Educación, 2010), así como a algunos centros que contaban con un número importante de alumnado extranjero ${ }^{3}$.

Especialmente el primer criterio mencionado ha sido muy importante

3 Debido a la reciente incorporación del/a Educador/a Social en los centros, y la poca familiarización con las funciones a realizar, en algunos casos han participado en la investigación otros agentes educativos que formaban parte de los Equipos de Orientación Educativa, como orientadores/as, pedagogos/as y trabajadores/as sociales, aunque en un porcentaje muy reducido. 
a la hora de seleccionar la muestra porque permite tener una visión global de nuestro objeto de estudio, sin que haya interferido preferencias o grado de accesibilidad a los mismos. Hemos de indicar que de los/as 66 educadores/as sociales que se han incorporado a los centros escolares andaluces en el curso 2009-2010, un total de 37 han cumplimentado los cuestionarios.

Los datos obtenidos se han tratado con el programa SPSS (Statistical Package for the Social Sciences).

\section{Resultados y discusión}

\subsection{La atención a la diversidad cultural: acciones, medidas y recursos}

Para profundizar en el desarrollo de actuaciones de los centros educativos en Andalucía para atender a la diversidad cultural, a continuación se presentan los resultados acerca de las medidas que se llevan a cabo, así como acerca de los/as profesionales cuya labor se relaciona directamente con estas tareas.

En primer lugar, en el $81.1 \%$ de los casos, las personas destinatarias respondieron que el enfoque intercultural se encuentra presente entre los objetivos del Plan Anual del Centro. En este sentido, es cierto que la interculturalidad aparece como finalidad del centro, pero se pueden encontrar situaciones contradictorias a esta filosofía en el momento en el que se analizan las prácticas concretas que se llevan a cabo, como se puede observar en algunos de los resultados que se exponen con posterioridad.

Ello puede conducir a reflexionar que, en muchos casos, la interculturalidad es un modelo de gestión de la diversidad que a nivel teórico se contempla como necesario y posible, aunque a la hora de llevarlo a la intervención directa, los criterios a seguir sean fácilmente confundidos con otros modos de atención a la diversidad cultural. Además, es Ilamativo el porcentaje de profesionales que no saben /no contestan esta cuestión $(16.2 \%)$, corroborando que no es un término identificable con las actuaciones de los centros. 
La diversidad cultural desde la perspectiva de los/as educadores/as sociales: un estudio en los centros educativos de Andalucía

Teresa Terrón-Caro, Teresa Rebolledo Gámez, M. ${ }^{a}$ del Rocío Rodríguez Casado y Macarena ESTEBAN IBÁÑ̃z

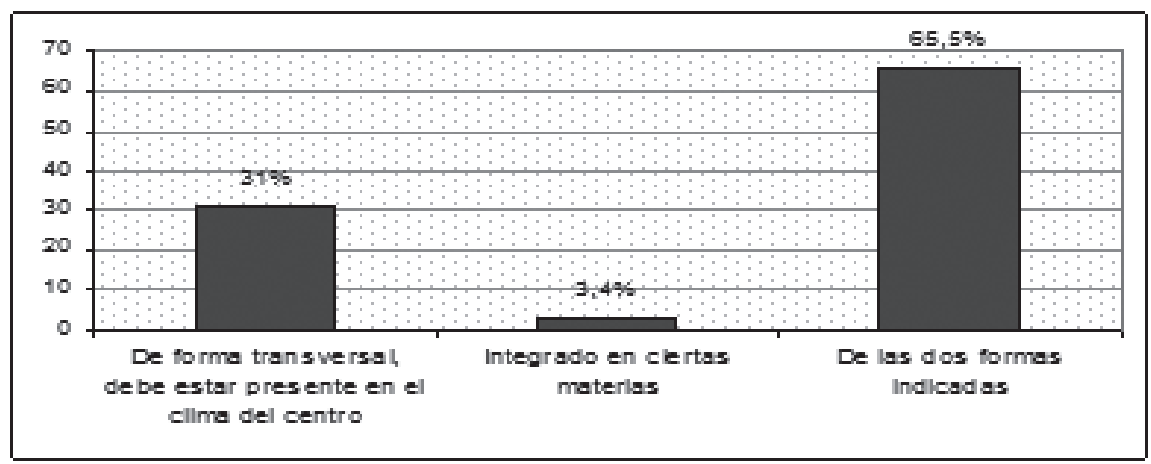

Figura 4. Posición que ocupa el enfoque intercultural en los documentos oficiales del centro

Analizando esta cuestión, de las personas que afirmaron que el centro poseía un enfoque intercultural en su planificación, el 31\% contestó que la interculturalidad aparecía de forma transversal en los documentos oficiales, ya que debía estar presente en el clima del centro, frente a un $3.4 \%$ de casos en los que se opinaba que este enfoque existía de manera integrada en materias específicas. Cabe decir que el $65.5 \%$ de los destinatarios/as respondieron que esta filosofía intercultural se encontraba en ambas opciones, tanto de forma transversal presente en el clima del centro como particular de algunas materias.

Concretando en las actuaciones específicas que existen para el alumnado de origen extranjero, se observan los resultados al planteamiento de la existencia de medidas de carácter curricular, pedagógico y organizativo que faciliten el acceso, la permanencia y la promoción educativa de dicho alumnado.

Con respecto a este tipo de medidas, el $82.9 \%$ de los/s profesionales aseguraron que su centro contaba con este tipo de acciones para atender a la diversidad cultural. Una de las opciones donde estas actuaciones pueden recogerse son en los Planes de Compensación Educativa, regidos por el Decreto 167/2003, de 17 de junio, el cual determina una serie de medidas de carácter compensador dirigidas a alumnado que, por diversas circunstancias personales o sociales se encuentra en situación de desventaja para el acceso, permanencia y promoción en el sistema educativo. En concreto, el artículo 9 de esta normativa establece que los centros escolares con este tipo de alumnado podrán elaborar un Plan de Compensación Educativa con la finalidad de adaptar el currículo a las necesidades y características del mismo. 
Teresa Terrón-Caro, Teresa Rebolledo Gámez, M. ${ }^{a}$ Del Rocío Rodríguez Casado y Macarena

En esta línea, los resultados de este estudio aportan que, en el $77.8 \%$ de los casos los centros educativos tenían un Plan de Compensación Educativa. Como apunte relevante, se debe considerar que los centros que tengan estos Planes deben guiarse por la Orden de 26 de febrero de 2004, en la que se regula por parte de la administración educativa, el procedimiento para la elaboración, la aprobación, aplicación y evaluación de estos planes compensatorios, así como también se normaliza su estructura, contenido y recursos adicionales, que sean necesarios para su desarrollo, por los centros docentes sostenidos con fondos públicos.

Para dilucidar la forma en que las compensaciones educativas se desarrollan en los centros, se planteó si los centros las ponían en marcha en forma de actividades extraescolares y/o complementarias. En este caso, el $71.4 \%$ de los centros organizaban actividades complementarias o extraescolares que trabajaban la compensación educativa. Es significativo destacar que el $14.3 \%$ de los/as profesionales no supieran o no contestaran a esta cuestión. Para adentrarse en este hecho, se preguntó qué tipo de actividades se realizaban, en su caso. Ante esta cuestión, se desprende que las actividades que aparecían para la compensación educativa de forma complementaria y/o extraescolar, son variadas ${ }^{4}$ : refuerzo escolar y lingüístico $(58.3 \%)$, acompañamiento $(37.5 \%)$, talleres $(29.2 \%)$, actividades deportivas $(16.7 \%)$ y salidas $(12.5 \%)$.

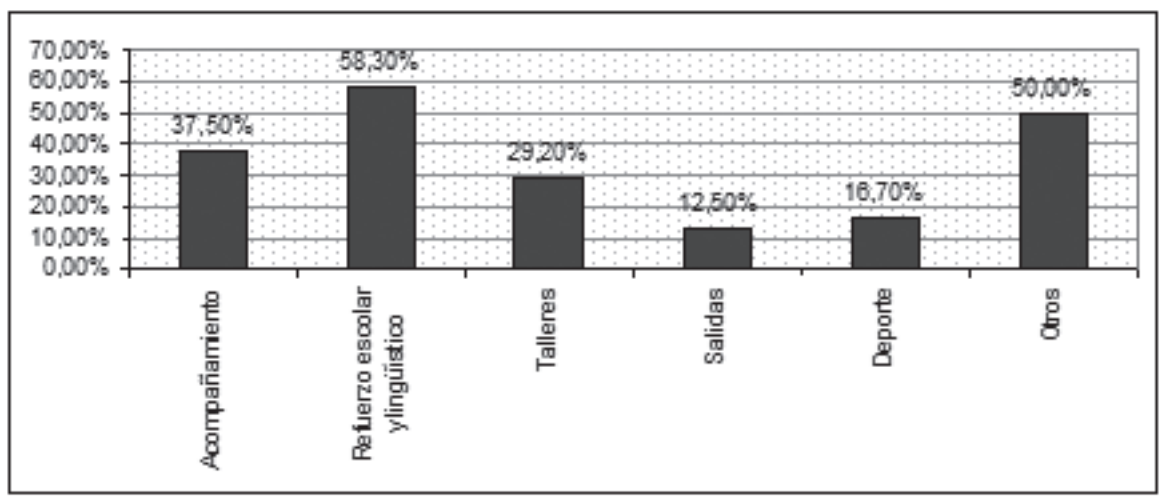

Figura 5. Actividades de compensación educativa programadas.

4 El porcentaje total de personas que respondieron a esta pregunta es superior al $100 \%$ debido a que se trata de una pregunta abierta de respuesta múltiple. 
La diversidad cultural desde la perspectiva de los/as educadores/as sociales: un estudio en los centros educativos de Andalucía

Teresa Terrón-Caro, Teresa Rebolledo Gámez, M. ${ }^{a}$ del Rocío Rodríguez Casado y Macarena Esteban IBÁÑez

Por otro lado, una de las actuaciones más extendidas para atender a alumnado de origen extranjero son las Aulas Temporales de Adaptación Lingüística (ATALs), que se definen como programas de enseñanza y aprendizaje del español como lengua vehicular, vinculado a profesorado específico, que permiten la integración del alumnado inmigrante en el centro y su incorporación a los ritmos y actividades de aprendizaje propios del nivel en el que se encuentren escolarizados atendiendo a su edad y a su competencia curricular (Orden de 15 de enero de 2007, por la que se regulan las medidas y actuaciones a desarrollar para la atención del alumnado inmigrante y, especialmente, las Aulas Temporales de Adaptación Lingüística).

El 71.4\% de los centros disponían de dichas ATALs como medidas de carácter compensatorio en relación al aprendizaje de la lengua española. En cambio, el $26.6 \%$ de los centros no desarrollaban esta medida, lo que puede explicarse por la poca o nula presencia de alumnado extranjero, ya que las ATALs suelen implantarse en centros que las demandan por necesidades de su alumnado.

Para comprender las actuaciones de atención a la diversidad cultural, es necesario conocer los recursos humanos especializados en este ámbito con los que cuentan los centros, pues supone uno de los aspectos fundamentales que puede incidir en el desarrollo de estas intervenciones. En este sentido, el $74.3 \%$ de los/as profesionales respondieron que la Consejería de Educación había adjudicado personal de apoyo para intervenir en la diversidad cultural de los centros.

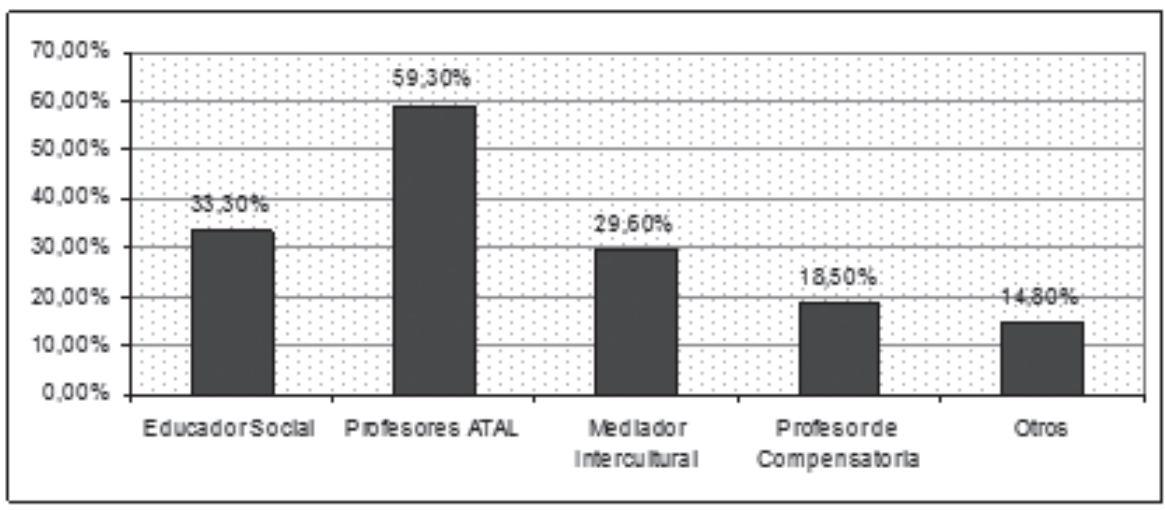

Figura 6. Tipología de profesionales para la atención a la diversidad cultural. 
De los datos obtenidos ${ }^{5}$ con respecto a la tipología de estos profesionales, se deduce que, en la mayoría de los casos (59.3\%), el/la profesional asignado era profesorado de ATAL, seguido de educadores/as sociales $(33.3 \%)$ y mediadores/as interculturales (29.6\%). Por último, se trataba de profesorado para la realización concreta de actividades de compensatoria $(18.5 \%)$.

$\mathrm{Si}$ bien cualquier profesional que atiende la diversidad cultural es sumamente importante para aumentar la calidad de la intervención que se dispense, consideramos interesante indagar si la figura del mediador o mediadora intercultural existía en el centro, como indicador de la relevancia que se le otorga a este tema. Según las respuestas de la muestra, sólo 4 de cada 10 centros disponían de profesionales de la mediación intercultural.

Un último aspecto referido a la intervención directa con el alumnado de origen extranjero se relaciona con las acciones específicas que el centro realiza para el desarrollo de la identidad cultural del alumnado perteneciente a grupos culturales minoritarios.

En cuanto a estas actuaciones, el $61.1 \%$ de los/as profesionales respondieron de forma afirmativa a la existencia de actividades dirigidas al desarrollo de la identidad cultural de este alumnado. De nuevo es Ilamativo que el $11.1 \%$ de las personas encuestadas no supieran o no contestaran a esta pregunta, ya que puede indicar que un alto número de personas no se encontraba con seguridad de distinguir el tipo de acciones que se orientan al desarrollo identitario del alumnado.

En los casos en los que estas actividades existían, en lo que respecta a los aspectos organizativos, se encuentran diferentes posibilidades: organizadas desde el centro escolar (90.5\%); organizadas por el profesorado $(4.8 \%)$; u organizadas en función de los ciclos educativos (4.8\%).

5 El porcentaje total de personas que respondieron a esta pregunta es superior al $100 \%$ debido a que se trata de una pregunta abierta de respuesta múltiple. 
La diversidad cultural desde la perspectiva de los/as educadores/as sociales: un estudio en los centros educativos de Andalucía

Teresa Terrón-Caro, Teresa Rebolledo Gámez, M. ${ }^{a}$ del Rocío Rodríguez Casado y Macarena ESTEBAN IBÁÑ̃z

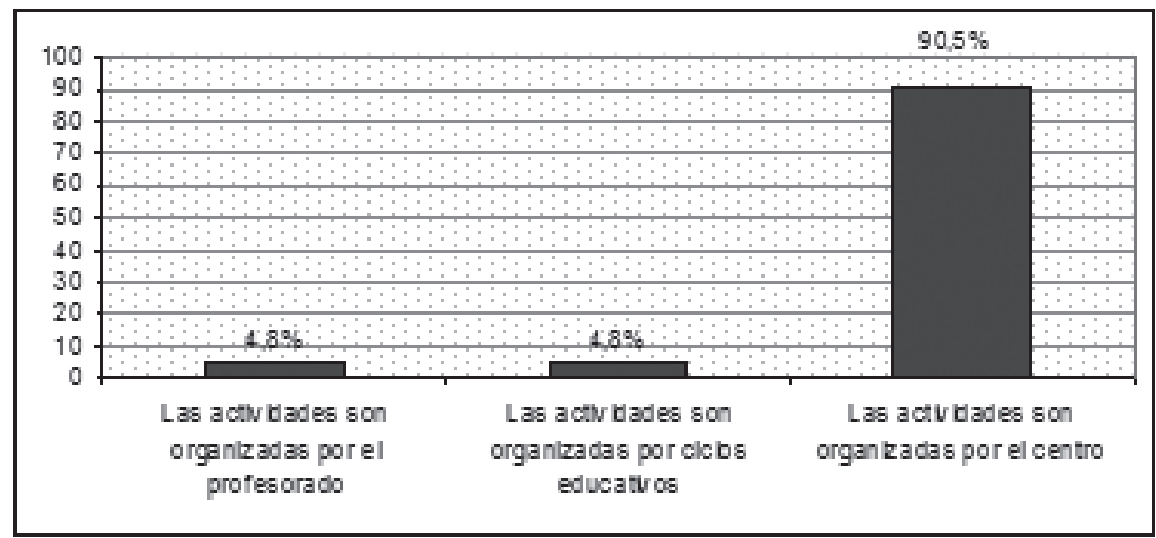

Figura 7. Tipos de organización de actuaciones para el desarrollo de la identidad cultural del alumnado perteneciente a grupos culturales no mayoritarios.

Finalmente, en los datos referidos a la frecuencia en que estas actividades se llevan a cabo, el $38.1 \%$ de los casos se realizaban de forma trimestral, seguido del $23.8 \%$ de los centros en los que se desarrollaban anualmente. Sólo el 19\% de las personas contestaron que se producían mensualmente $y$, en el mismo, porcentaje, semanalmente.

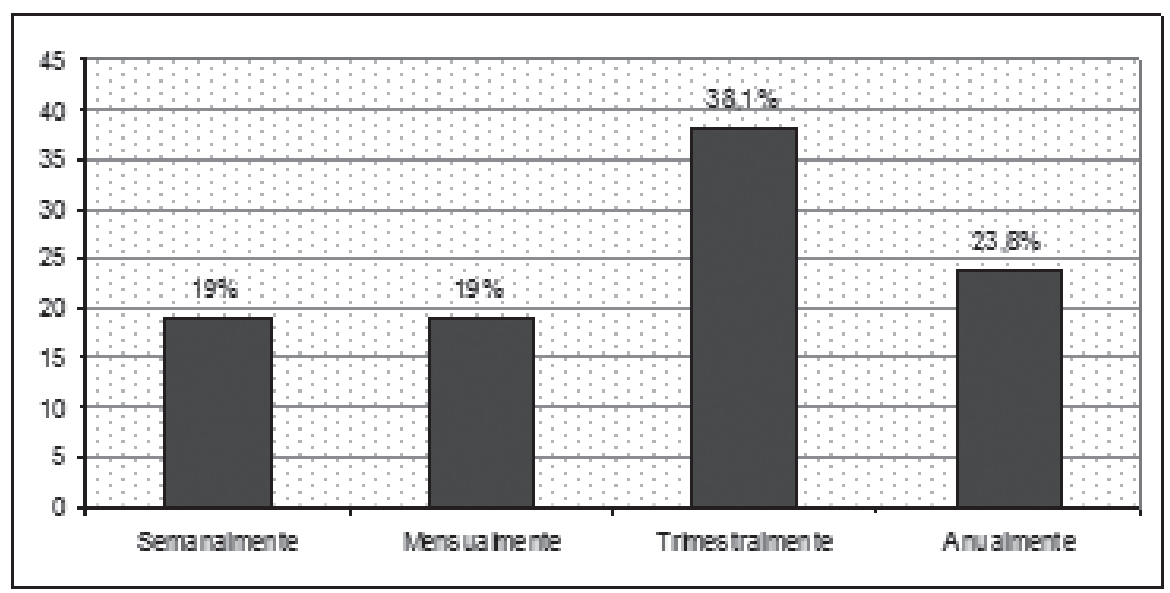

Figura 8. Frecuencia en la que se realizan las acciones específicas de desarrollo de la identidad cultural del alumnado perteneciente a grupos culturales no mayoritarios.

Este hecho se relaciona directamente con el enfoque intercultural que se da a las actividades, ya que según este modelo educativo, las acciones 
Teresa Terrón-Caro, Teresa Rebolledo Gámez, M. ${ }^{a}$ del Rocío Rodríguez Casado y Macarena

que atienden a la diversidad cultural no deben darse de forma puntual, como se deduce de los datos extraídos, sino que son actuaciones que se dan en la vida diaria de los centros, evitando la exaltación folclórica de las culturas que se produce al relacionar dichas intervenciones con hechos ocasionales en las instituciones educativas.

\subsection{Relaciones con el contexto: familia y agentes del entorno}

Otro de los elementos que ayuda a evidenciar el modelo de gestión de la diversidad que siguen los centros tiene que ver con las relaciones que se mantiene con los agentes de la comunidad educativa. Entre estos agentes, las familias se destacan como elementos indispensables para una adecuada atención a la diversidad dentro y fuera del espacio escolar.

Tomando como indicio las acciones directas que plantean los centros para facilitar las relaciones familias (de origen extranjero)-escuelas, se cuestiona si los centros disponían de información escrita en otros idiomas sobre los recursos propios. Sólo el $17.1 \%$ de las personas encuestadas afirmaban la existencia de estas medidas, mientras que la mayoría (82.9\%) no ofrecían este recurso. Esta falta de información adaptada a otros idiomas, evidenciaría que existen auténticos obstáculos que dificultan una comunicación fluida con las familias, así como impide la inclusión real de familias que no hablan y/o leen en español en los centros donde sus hijos o hijas se encuentran matriculados.

Otro de los recursos con los que los centros pueden contar para apoyar la participación de las familias de origen extranjero en los centros escolares, sobre todo, aquellas que no hablan el idioma autóctono, lo constituyen los/as intérpretes. Planteando si se disponían de intérpretes en las primeras reuniones con las familias que no hablaran español, el $85.7 \%$ de los centros no contaban con este tipo de profesional. Con este dato, se vuelve a incidir en la escasa presencia de recursos básicos que fomenten la implicación de las familias en mayor desventaja por desconocimiento del idioma.

Por último, cabe decir que el buen funcionamiento de la atención a la diversidad cultural en las escuelas puede ser determinado por el contexto donde se ubiquen las intervenciones. Dentro de los procedimientos a seguir, un aspecto relevante es la coordinación entre los agentes del entorno en el que se enmarca la escuela.

En este sentido, el $71.4 \%$ de los/as profesionales defendieron que 
La diversidad cultural desde la perspectiva de los/as educadores/as sociales: un estudio en los centros educativos de Andalucía

Teresa Terrón-Caro, Teresa Rebolledo Gámez, M. ${ }^{a}$ del Rocío Rodríguez Casado y Macarena Esteban IBÁÑez

existía coordinación entre las administraciones públicas, asociaciones y organizaciones no gubernamentales en el desarrollo de las actuaciones de atención a la diversidad que se desarrollaban, mientras en el $28.6 \%$ de los casos, esta coordinación era nula.

\section{Conclusiones}

Se puede concluir que los resultados expuestos en este trabajo muestran varios aspectos fundamentales que debemos tener en cuenta al discernir sobre las acciones educativas para la atención a la diversidad en los centros escolares: por un lado, es necesario destacar que el perfil profesional de las personas que son responsables de las acciones en diversidad cultural es heterogéneo y difícil de concretar, ya que son muchos los tipos de profesionales que forman parte de estas acciones; y, por otro lado, se debe tener presente que la realidad de la atención a la diversidad cultural en las escuelas andaluzas es compleja, en tanto las medidas son variadas y no atienden a un modelo específico de gestión de la diversidad.

En referencia a la primera consideración planteada, se parte de una situación actual en la que los/as educadores/as sociales se están incorporando progresivamente al contexto escolar, adoptando un papel cada vez más relevante en las acciones que las escuelas Ilevan a cabo para el fomento de una convivencia en términos de diversidad.

A pesar de ello, no se puede olvidar que, cuando se habla de atención a la diversidad cultural en las escuelas, es imprescindible contemplar las actuaciones desde una perspectiva multi/inter-disciplinar, como es el caso de los EOEs, donde todo profesional puede realizar aportaciones que, en conjunto, otorguen beneficios en cada una de las acciones a desarrollar.

Con respecto al enfoque de atención a la diversidad cultural en los centros educativos de Andalucía, las acciones que dichas instituciones realizan en materia de diversidad apuntan a la presencia de distintos modelos de gestión de la diversidad.

Por un lado, se encuentran acciones que pueden relacionarse con un modelo intercultural, tales como la existencia de un enfoque intercultural en los Planes Anuales del Centro, el trabajo de la interculturalidad de forma transversal y específica, medidas curriculares, pedagógicas y organizativas para promocionar la permanencia del alumnado de origen 
Teresa Terrón-Caro, Teresa Rebolledo Gámez, M. ${ }^{a}$ del Rocío Rodríguez Casado y Macarena

extranjero o la coordinación entre diferentes organismos en las medidas de atención a la diversidad cultural.

Este tipo de intervenciones favorece que la interculturalidad se encuentre presente en la filosofía del centro, de manera que los/as profesionales puedan contextualizar sus acciones en un marco de convivencia entre culturas y fomento de la igualdad entre el alumnado, independientemente de su procedencia étnica o cultural.

Sin embargo, son varios los indicadores que muestran que se llevan a la práctica procedimientos que poco tienen que ver con un modelo de gestión intercultural. Muchos centros educativos poseen Planes de Compensación Educativa, acompañados por actividades extracurriculares que se orientan en esta línea. Este hecho, junto con la existencia de actividades dirigidas específicamente al alumnado de origen extranjero o perteneciente a culturas minoritarias, organizadas por el centro y de forma puntual, son acciones que se acercan a modelos compensatorios o multiculturales, en los que la diversidad cultural se entiende como necesidad educativa que requiere de medidas especiales dirigidas a un grupo de alumnado concreto que se encuentra en situación de desventaja con respecto al resto.

Las ATALs son un ejemplo de ello, ya que se convierten en instrumentos de las escuelas para apoyar la integración del alumnado extranjero a través del aprendizaje del idioma autóctono, a la vez que separa al alumnado del discurrir normalizado del aula en determinados periodos del ciclo escolar.

En cuanto a las relaciones con las familias, las acciones del centro también evidencian la escasa adaptabilidad a las situaciones de diversidad cultural, no contemplando la existencia de información básica de la escuela en otros idiomas o no contando con intérpretes en las reuniones con familiares de habla no hispana.

En conclusión, esta realidad lleva a confirmar que, al respecto de la atención a la diversidad cultural, los centros aún no muestran referentes claros de un modelo específico, tomando elementos de distintos modelos en la filosofía de la intervención.

Por ello, frente a prácticas que favorecen la segregación del alumnado, las actividades compensatorias o aspectos de una gestión multicultural, se debe defender, no sólo la introducción de la perspectiva intercultural a nivel teórico, sino la puesta en práctica de un modelo educativo que promueva 
La diversidad cultural desde la perspectiva de los/as educadores/as sociales: un estudio en los centros educativos de Andalucía

Teresa Terrón-Caro, Teresa Rebolledo Gámez, M. a del Rocío Rodríguez Casado y Macarena ESTEBAN IBÁÑ̃z

(...) estrategias y actitudes inclusivas en las que tanto el alumnado inmigrante como el autóctono mantengan los valores propios de su cultura de origen, a la vez que valore, respete y adopte los de otras. Se inicia así un proceso de convivencia y aprendizaje mediante la interacción y el enriquecimiento mutuo de todas las lenguas y culturas que permite que a través del desarrollo de la identidad individual de cada alumna o alumno se conforme también una identidad grupal (Junta de Andalucía, 2011).

En definitiva, se trata de actuar en base a la lucha contra las desigualdades, la participación de la comunidad educativa y la inclusión de alumnado, sin diferencias por etnia, cultura o religión, avanzando, de esta manera, hacia una mejora de la educación que nos concierne a todas y todos.

\section{Referencias bibliográficas}

AGUADO ODINA, T. (2003). Pedagogía intercultural. Madrid: McGraw-Hill.

AGUADO ODINA, T. (2004). Investigación en educación intercultural. Educatio Sig/o $X X I, 22,39-57$.

ÁLVAREZ CASTILLO, J. L. (2008). La investigación en educación intercultural. En PANTOJA, A., CAMPOY, T.J., JIMÉNEZ, A. Y VILLANUEVA, C. (Coord.). El carácter universal de la educación intercultural. Actas de las I Jornadas Internacionales y VI sobre Diagnóstico y Orientación. Jaén: Servicio de Publicaciones de la Universidad. Recuperado de http://www.ujaen.es/investiga/ideo/jornadas/recursos/actasConISBN.pdf

ARIÑO VILLARROYA, A. (2009). Estilos de aculturación y encrucijadas de la diversidad cultural. Papers, 94, 115-137.

BARANDICA PAIRET, E. (2006). Educación y multiculturalidad: análisis, modelos y ejemplos de experiencias escolares. En M. A. ESSOMBA (Coord.). Construir la escuela intercultural. Reflexiones y propuestas para trabajar la diversidad étnica y cultural. Barcelona: Graó.

BARTOlOMÉ PINA, M. (COORD.) et al (1997). Diagnóstico a la Escuela Multicultural. Barcelona: Cedecs Editorial.

BESALÚ COSTA, X. (2002). Diversidad cultural y educación. Madrid: Síntesis Educación.

JUNTA DE ANDALUCÍA, CONSEJERIA DE EDUCACIÓN (2010). Instrucciones de la Dirección General de Participación e Innovación Educativa por las que se regula la intervención del educador y educadora social en el ámbito educativo.

DE MIGUEL BADESA, S. (1995). Educación, escuela y formación del educador para una sociedad inter-multicultural. Tendencias Pedagógicas, 2, 43-53.

DECRETO 167/2003, DE 17 DE JUNIO, POR EL QUE SE ESTABLECE LA ORDENACIÓN DE LA ATENCIÓN EDUCATIVA A LOS ALUMNOS Y ALUMNAS CON NECESIDA- 
La diversidad cultural desde la perspectiva de los/as educadores/as sociales: un estudio en los centros educativos de Andalucía

Teresa Terrón-Caro, Teresa Rebolledo Gámez, M. ${ }^{a}$ Del Rocío Rodríguez Casado y Macarena

ESTEBAN IBÁÑEZ

DES EDUCATIVAS ESPECIALES ASOCIADAS A CONDICIONES SOCIALES DESFAVORECIDAS. BOJA n 118 (2003).

DECRETO 213/1995, DE 12 DE SEPTIEMBRE DE 1995, POR EL QUE SE REGULAN LOS EQUIPOS DE ORIENTACIÓN EDUCATIVA. BOJA n ${ }^{\circ} 153$ (1995).

GARCÍA CASTAÑO, F.J., RUBIO GÓMEZ, M., Y BOUACHRA, O. (2008). Población inmigrante y escuela en España: Un balance de investigación. Revista de Educación, 345, 23-60.

GIL-JAURENA, I. (2013). Enfoque intercultural y animación sociocultural: convergencias y reflexiones. Revista Iberoamericana de Educación, 61/4. Recuperado de http:// www.rieoei.org/deloslectores/5404Gil.pdf

JUNTA DE ANDALUCÍA (2011). Guía Básica de Educación Intercultural. España: Junta de Andalucía.

LEY 17/2007, DE 10 DE DICIEMBRE, DE EDUCACIÓN DE ANDALUCÍA. BOJA nº 252 (2007).

MINISTERIO DE EDUCACIÓN, CULTURA Y DEPORTE (2014). Enseñanzas no universitarias. Alumnado matriculado. Curso 2013-2014. Datos avance. Recuperado de http://www.mecd.gob.es/servicios-al-ciudadano-mecd/estadisticas/educacion/nouniversitaria/alumnado/matriculado/2013-2014--Datos-Avances.html

MINISTERIO DE EDUCACIÓN, CULTURA Y DEPORTE (2014). Extranjeros con certificado de registro o tarjeta de residencia en vigor. Recuperado de http://extranjeros. empleo.gob.es/es/Estadisticas/operaciones/con-certificado/index.html

ORDEN DE 15 DE ENERO DE 2007, POR LA QUE SE REGULAN LAS MEDIDAS Y ACTUACIONES A DESARROLLAR PARA LA ATENCIÓN DEL ALUMNADO INMIGRANTE Y, ESPECIALMENTE, LAS AULAS TEMPORALES DE ADAPTACIÓN LINGÜÍSTICA. BOJA n 33 (2007).

ORDEN DE 23 DE JULIO DE 2003, POR LA QUE SE REGULAN DETERMINADOS ASPECTOS SOBRE LA ORGANIZACIÓN Y EL FUNCIONAMIENTO DE LOS EQUIPOS DE ORIENTACIÓN EDUCATIVA. BOJA n 155 (2003).

ORDEN DE 26 DE FEBRERO DE 2004, EN LA QUE SE REGULA POR PARTE DE LA ADMINISTRACIÓN EDUCATIVA, EL PROCEDIMIENTO PARA LA ELABORACIÓN, LA APROBACIÓN, APLICACIÓN Y EVALUACIÓN DE ESTOS PLANES COMPENSATORIOS, ASÍ COMO TAMBIÉN SE NORMALIZA SU ESTRUCTURA, CONTENIDO Y RECURSOS ADICIONALES, QUE SEAN NECESARIOS PARA SU DESARROLLO, POR LOS CENTROS DOCENTES SOSTENIDOS CON FONDOS PÚBLICOS. BOJA, n 52 (2004).

ORTEGA, I (2004). Orientaciones para la Elaboración del Plan de Acogida del alumnado inmigrante. Victoria-Gasteiz: Servicio Central del Gobierno Vasco. Recuperado de http://www.aulaintercultural.org/article.php3?id_article=1090

OSUNA NEVADO, C. (2012). En torno a la educación intercultural. Una revisión crítica. Revista de Educación, 358. Recuperado de http://www.uned.es/grupointer/re358_03_ carmen_osuna.pdf

PLAN ESTRATÉGICO DE CIUDADANÍA E INTEGRACIÓN (2007 - 2010). Ministerio de Empleo y Seguridad Social. Secretaria General de Inmigración y Emigración. Recuperado de http://extranjeros.empleo.gob.es/es/IntegracionRetorno/Plan_estrategico/ 
La diversidad cultural desde la perspectiva de los/as educadores/as sociales: un estudio en los centros educativos de Andalucía

Teresa Terrón-Caro, Teresa Rebolledo Gámez, M. ${ }^{a}$ del Rocío Rodríguez Casado y Macarena ESTEBAN IBÁÑEZ

RODRÍGUEZ NAVARRO, H., GALLEGO LÓPEZ, B., SANSÓ GALIAY, C., NAVARRO SIERRA, J. L., VELICIAS SÁNCHEZY M., LAGO SALCEDO, M. (2011). La educación intercultural en los centros escolares españoles. Revista Electrónica Interuniversitaria de Formación del Profesorado-REIFOP, 14 (1), 101-112.

RODRÍGUEZ VARGAS A. (2012). Modelos de gestión de la diversidad cultural y la integración escolar del alumnado inmigrado. Revista Educación Inclusiva, 5, (3), 47-62.

SÁEZ CARRERAS, J. Y CAMPILLO DÍAZ, M. (2013). La Pedagogía Social como comunidad disciplinar: entre la profesionalización y desprofesionalización del campo. Educatio Siglo XXI, 3 (2), 73-96.

SCHRAMKOWSKI, B. (2008). Una actitud crítica frente al racismo como fundamento de las competencias interculturales. En PANTOJA, A., CAMPOY, T.J., JIMÉNEZ, A. Y VILLANUEVA, C. (Coord.). El carácter universal de la educación intercultural. Actas de las I Jornadas Internacionales y VI sobre Diagnóstico y Orientación. Jaen: Servicio de Publicaciones de la Universidad. Recuperado de http://www.ujaen.es/investiga/ ideo/jornadas/recursos/actasConISBN.pdf

TERRÓN CARO, M.T. Y CÁRDENAS RODRÍGUEZ, R. (2012). Planificar por competencias con enfoque intercultural. Escuela Abierta, 15, 33-50. 